\title{
PTU-003 CAMPYLOBACTER COLITIS COMPLICATED BY TOXIC MEGACOLON: PREVALENCE, CHARACTERISTICS AND OUTCOME
}

doi:10.1136/gut.2011.239301.131

E L Culver," E Morris, P Trivedi, A J Ellis Department of Gastroenterology, Horton Hospital, Banbury, UK

Introduction Campylobacter gastroenteritis is usually a self limiting infection; the development of colitis and toxic megacolon are rare complications that may mimic inflammatory bowel disease (IBD). This study aimed to define the prevalence, characteristics and outcome of patients with campylobacter colitis and toxic megacolon in our institution.

Methods A retrospective review of medical notes, laboratory data, imaging and endoscopic records for adult inpatients with documented diarrhoea and campylobacter detected in stool cultures, over a five-year period was performed. Colitis was defined as the presence of mucosal inflammation at endoscopy with compatible histology and/or abdominal imaging consistent with mucosal oedema. Toxic megacolon was defined as non-obstructive colonic dilatation greater than $6 \mathrm{~cm}$ with signs of systemic toxicity. The R statistical package was used for regression analysis with log transformations and generalised linear model with binary response.

Results Eighty-seven patients had a positive stool culture for campylobacter, of which 56 were admitted to hospital. Fifteen patients $(27 \%)$ had evidence of colitis, and one-third of these developed features of toxic megacolon. All patients had a high CRP at presentation (median 111; range $12-215 \mathrm{mg} / \mathrm{dl}$ ). The diagnosis of colitis was associated with the presence of blood in the stool $(p=0.05)$, but independent of age and other clinical and laboratory markers of severity. The median duration to diagnosis of colitis and megacolon was 5 (1-43) and 6 (6-21) days, respectively. The median duration of diarrhoea for uncomplicated infection, colitis and megacolon was 5 (1-36), 10 (1-28) and 15 (5-28) days, respectively. Length of admission was dependent upon age $(p=0.03)$ and duration of diarrhoea $(p$ $=0.00001$. .

Patients with colitis were more likely to require surgical review $(p=0.02)$ and be treated with ciprofloxacin $(p=0.04)$. IBD was more likely to be a differential diagnosis in those with colitis $(p=0.001 ; O R$ 16.5) and in younger patients ( $p=0.03$; OR 1.7). No patient had co-existent IBD, although 1 patient $(1.7 \%)$ was diagnosed with Crohn's colitis one year later. No patient perforated or required colectomy.

Conclusion Colitis and toxic megacolon occurred in $27 \%$ and $9 \%$ of patients admitted to hospital with campylobacter in stool cultures, respectively. One-third of patients with colitis progressed to toxic megacolon. All patients had a high CRP on admission, the level of which may help to distinguish them from patients with IBD. Early stool culture is essential to guide diagnosis and management. Outcome appears more favourable compared to megacolon complicating IBD in this cohort.

Competing interests None.

Keywords campylobacter, colitis, toxic megacolon. 